\title{
A New Ant Colony Algorithm for Deep Sea Sparse Network
}

\author{
Hongning $\mathrm{Hu}^{1,}$ a, b, Zixi Tian² \\ ${ }^{1}$ Naval University of Engineering, Wuhan 430033, China; \\ ${ }^{2}$ The Naval Gun System Military Agent's Room in Shanghai Area, Shanghai 200135, China. \\ asynorsmith@hotmai.com, bhuhongning@hotmail.com
}

\begin{abstract}
Inspired by the biological habits of ants, the paper proposes a new routing algorithm for deep sea sparse networks without geographical location information. After absorbing the essence of the traditional ant colony algorithm, the new algorithm introduces the "smell" pheromone into the path construction. The simulation results show that the new algorithm can solve the problem of finding a path in deep sea sparse network, and will have a perfect application prospect.
\end{abstract}

Keywords: Ant colony algorithm; "Smell” pheromone; Deep sea sparse network.

\section{Introduction}

The directional flooding routing algorithms based on geographical location information can solve the problem [1] of underwater acoustic network, and will also increase the network load. Moreover, the algorithms often fails when the network is sparse [2], and it is difficult for the current technology to obtain the location information of nodes in deep sea. Therefore, it is urgent to find a new algorithm to replace the directional flooding algorithms which will maintain or even increase the performance without using nodes' location information.

The ant colony algorithm [3] has achieved remarkable achievements [4 7] in seeking the solution of continuous practical problems. The most domestic representative are the multi objective ant colony algorithm[8] proposed by Zhang Yongde, and the ant colony algorithm based on the foraging and returning mechanism, which is proposed by Kim Hao MO-FHACO[9]. The pheromone is released by ants in MO-FHACO's mechanism. Although this mechanism can better solve some continuous domain multi-objective optimization problem, the convergence of the algorithm takes a long time due to the insufficient pheromone in initial stage. Therefore, ant colony algorithm is difficult to be directly applied to sparse deep ocean self-organizing network.

This paper is inspired by the biological process of ant colony foraging, and proposes a new Pheromone update mechanism, and proposes a new deep sea routing algorithm based on this mechanism. The algorithm can solve the "hole" problem [2, 10] widespread in the sparse network, and is gradually convergent to the current optimal path with the increase of the number of packet transmission.

\section{A new ant colony algorithm for deep sea sparse network}

\subsection{Basic principles}

The smell of food, which plays a decisive role in the early stage of the ant's search for food. Inspired by the biological characteristics, the new algorithm introduces the "smell" pheromone into the pheromone update mechanism in the ant colony algorithm. Experimental results show that this mechanism ensures that the algorithm can quickly find the appropriate transmission path in deep sea sparse network.

Deep sea network consists of two kind of nodes which are super-nodes and normal-nodes [2, 10]. The normal-node constitute the main body of the deep sea network, and is responsible for the transmission of packets. The super-node is responsible for the generation and reception of information, it has two states of "food" and "nest". The basic principles of the new algorithm are as follows: 
(1) When super-node is in the "food" state, it broadcasts the "smell" pheromone in each interval of time. The unique identity of super-node and last-hop node, and current number of hops are contained in "smell" pheromone. The concentration of "scent" is determined by the number of hops taken, which means that the more hops, the lower the "smell" concentration;

(2) When it meets some communication needs, super-node immediately turns into the "nest" state, and then releases "ants" looking for a path from the "nest" to "food" by using "smell" and "ant" pheromone as conditions.

\subsection{Pheromone update mechanism}

In the algorithm, the pheromone contains "smell" pheromone and "ant" pheromone, whose update mechanism is described as follows:

(1) The update mechanism of "smell" pheromone is given in the formula (1).

$\eta(k) \leftarrow\left(1-\frac{k}{L}\right) \times \Gamma_{\text {food }}$

Among them, $\Gamma_{\text {food }}$ is "smell" pheromone of the target super-node; L means the maximum number of hops, which is consistent with the laws of nature; $\mathrm{k}$ represents the number of hops required to reach the present node from the target super-node;

(2) The update mechanism of "ant" pheromone is divided into global pheromone update mechanism and local pheromone update mechanism. Among them, the global pheromone update mechanism uses the ant colony system (ACS) [11] update mechanism. That is, after each iteration, only one ant (up to the best ant) is allowed to release the pheromone, as shown in the formula (2):

$\tau_{i j} \leftarrow(1-\rho) \tau_{i j}+\frac{\rho}{C^{b s}} \quad, \quad \forall(i, j) \in T^{b s}$

Wherein, $C^{b s}$ represents the length of the path traversed by the ants. $\rho$ has value of 0.5 in the experiment.

According to the formula (3), the new algorithm modifies the local update mechanism of ACS.

$\tau_{i j} \leftarrow(1-\varepsilon) \tau_{i j}+\Delta \tau_{0} \quad, \quad 0<\varepsilon \leq 1$

Among them, $\varepsilon$ is the evaporation coefficient, and the value is $0.1 ; \Delta \tau_{0}$ is the pheromone of ants, and the value is 0.3 . These two values are constant. At the initial time, the value of the "ant" pheromone in all nodes is 0 .

(3) An ant in the node $\mathrm{i}$, according to the pseudo random proportion rule, selects a node $\mathrm{j}$ as the next hop node from neighbor nodes. This rule is as follows:

$$
j=\left\{\begin{array}{lc}
\arg \max \left\{\tau_{i l}\left[\eta_{i l}\right]^{\beta}\right\} & q>q_{0} \\
\frac{\left[\tau_{i j}\right]\left[\eta_{i j}\right]^{\beta}}{\sum_{l \in N_{i}^{c}}\left[\tau_{i l}\right]\left[\eta_{i l}\right]^{\beta}} & q \leq q_{0}
\end{array}\right.
$$

Here, $q$ is a random numerical value in $[0,1]$, and $q_{0}=0.5$.

\subsection{Algorithm flow}

(1) At the beginning, all super-nodes are in the "food" status, and broadcast their own "smell" pheromone at set intervals. After receiving the "smell" message from a certain super-node, nodes in the network update and save the "smell" pheromone of the super-node, and then only forward it once;

(2) When a super-node meets some communication requirements who is assumed as the node S, it immediately changes it's status into the "nest" status, and sets the size of the ant colony, the maximum number of iterations, and initialize all ants' pheromone according to the total amount of packets;

(3) The target super-node who is the receiver of communication is called the node D. All ants select the next hop node from the neighbors according to the concentration of D's "smell" pheromone. When there are more than one node match the Highest concentration, Ants will randomly select a 
node as the next hop node among the highest concentration nodes, then put the present node into the collection of nodes arrived by the ants which is called the taboo set.

(4) For each ant do

While ( $\mathrm{D}$ is not an neighbor and there exist some neighbors not arrived by ant)

\{

a random number $\mathrm{x}$ has been generated between $[0,1]$;

if $x>0.5$

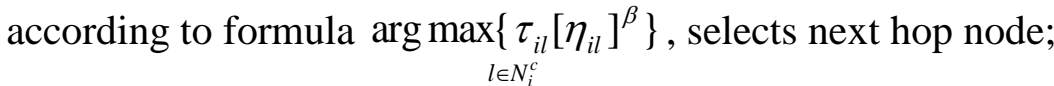

else

according to $P_{i j}^{k}(t)=\left\{\begin{array}{ll}\frac{\tau_{i j}^{\alpha}(t) \eta_{i j}^{\beta}(t)}{\sum_{s \in \text { allowed }_{k}} \tau_{i s}^{\alpha}(t) \eta_{i s}^{\beta}(t)} & \text { if } \quad j \in \text { allowed }_{k} \\ 0 & \text { otherwise }\end{array}\right.$,

Calculate the transition probability of each node, then select the maximum probability End of node as the next node, and add the node into ant's taboo set;

End

(5) Records and updates the optimal solution of this iteration;

(6) According to formula $\tau_{i j}(t+1)=(1-\rho) \cdot \tau_{i j}(t)+\sum \Delta \tau_{i j}{ }^{k}(t)$, updates the pheromone concentration of path.

(7) Sets $\mathrm{x}=\mathrm{x}+1$. If $\mathrm{x}<N_{\max }$, then jump into $\mathrm{step}(4)$; or the iteration will be recarried out until the status of $\mathrm{S}$ is retransformed into the "food" status.

\section{Simulation experiment}

The experiment fully adopts the network model and simulation parameters in the literature[2,10], except for that the number of normal-nodes have reduced from 400 to 200, as shown in figure 1 .

The DREAM is a typical flooding routing algorithm which has a certain representation, so this paper will use it as a reference to check the performance of the new algorithm. In order to give full play to the performance of DREAM, it is assumed that DREAM has been informed of the location information of all nodes.

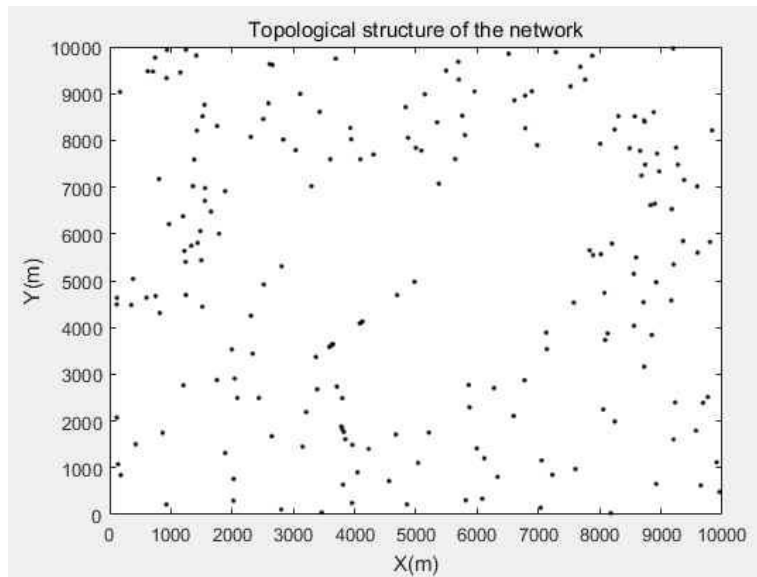

(a) Distribution of nodes in the network

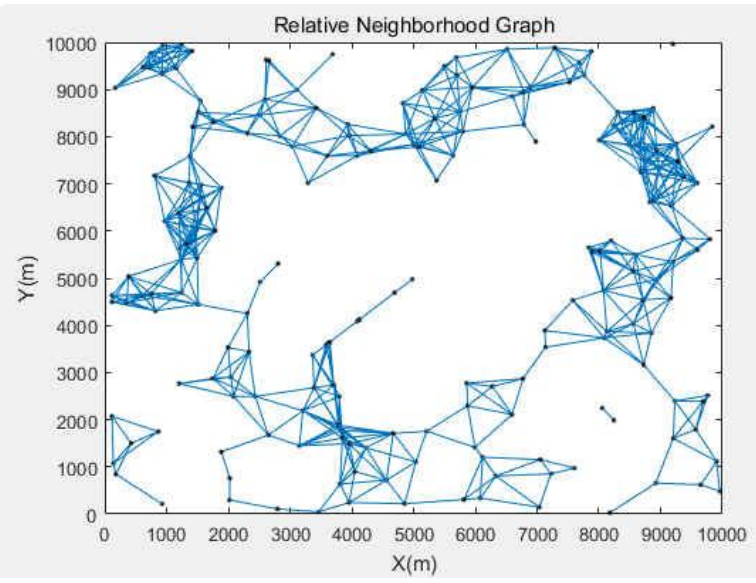

(b) Network connectivity

Fig. 1 Network Model in experiment

When the network is initialized, all super-nodes are in "food" state and spread the "smell" throughout the network. Taking the super-node in the upper right corner of network as an example, 
who is the "red +", figure 2 shows the diffusion of its "scent" pheromone, in which concentration from the high to the end and the color is changed from red to blue.

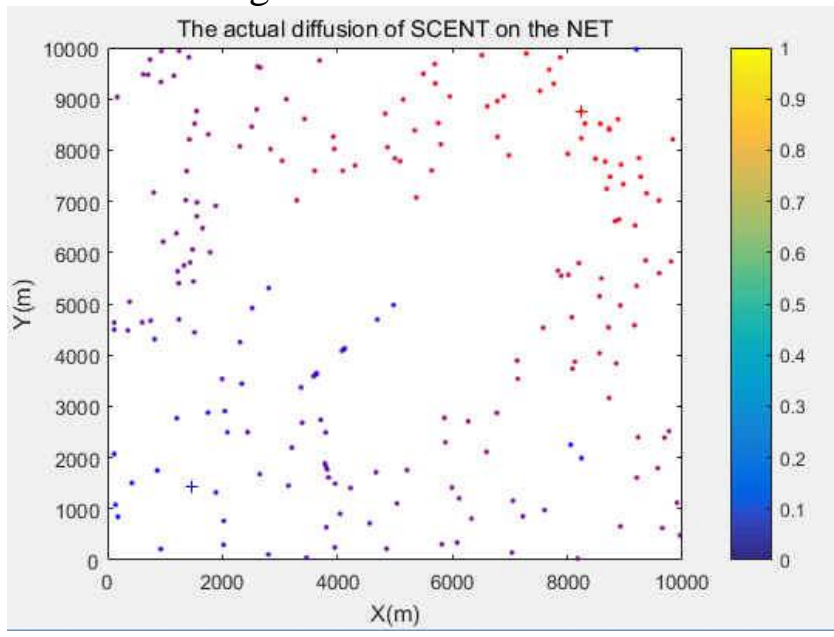

Fig 2 The diffusion of "smell" pheromone in the network

The experimental scenario is: a super-node "blue +" randomly sends 100 packets to another super-node "red +" within an hour.

The first, twentieth, fiftieth and 100th times of the new algorithm's data packet transmission are presented in figure 3. It is showed that under the dual influence of pheromone and ant pheromone, the new algorithm will be more and more convergent to the current optimal path with the increase of the number of communication.

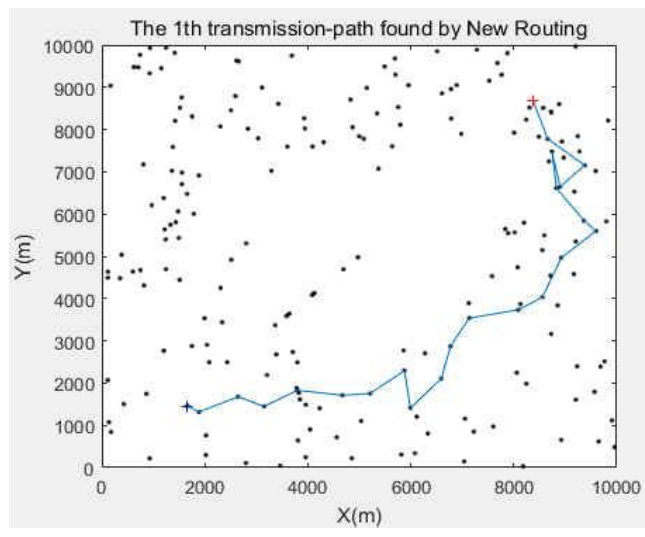

(a)The 1th transmission

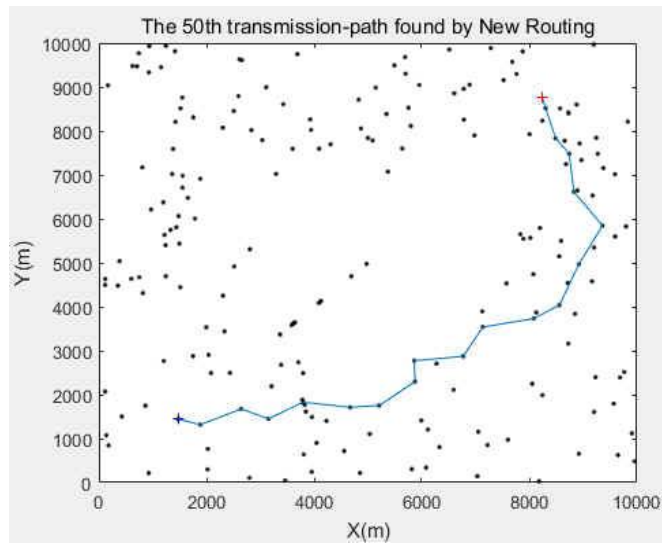

(c) The 50th transmission

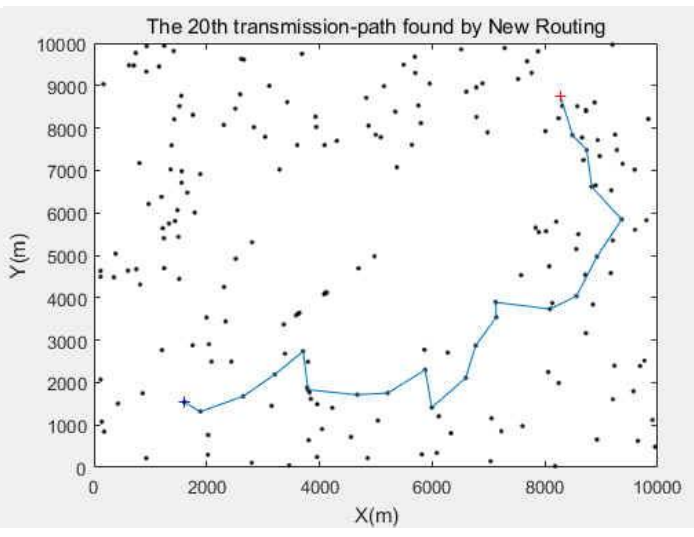

(b) The 20th transmission

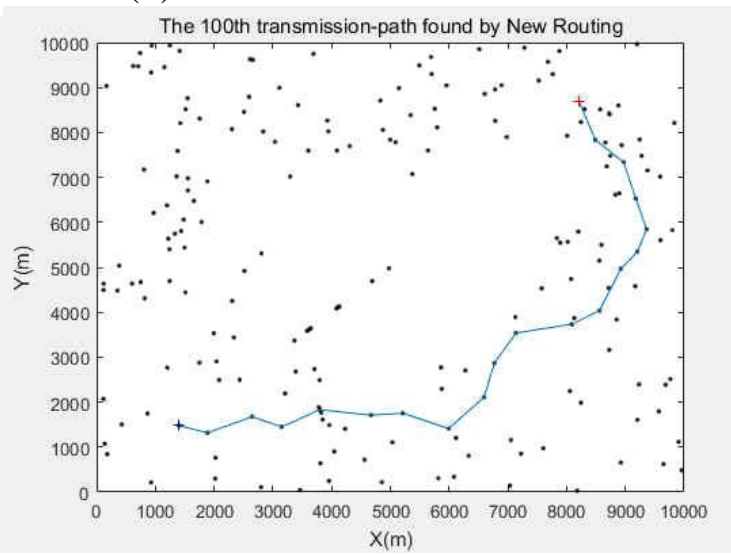

(d) The 100th transmission

Fig. 3 The actual propagation of the new algorithm

Figure 4 is the data packet transmission of DREAM algorithm. Because the network is sparse, the DREAM needs a large number of nodes joining to forward and retransmit packets, which also ensures packets could meet the target node successfully along multiple paths. DREAM has a good robustness, but also will cause a great waste of network energy and resources. At the same time, because every 
transmission of DREAM is an independent event, conditions of the path selection is almost unchanged, so the algorithm of each packet's transmission path is basically unchanged.

In the initial stage of the iteration of the new algorithm, ants select a path entirely dependent on "smell" pheromones, which attract some nodes participate into the process of selecting a path; At the late stage of iteration, the concentration of ant pheromone is proportional to the volatile coefficient, and the "smell" pheromone will not change in the whole communication process, which makes ants possible to explore a new path. The time delay and energy-consumed of the new algorithm presented in Figure 5 is a favorable proof of this "exploration" ability.

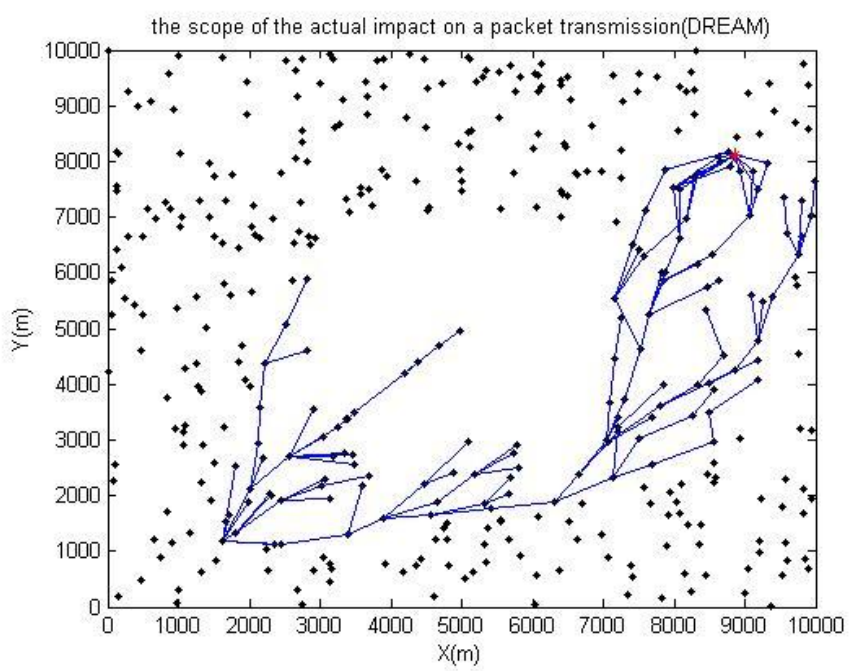

Fig. 4 The actual propagation of DREAM algorithm

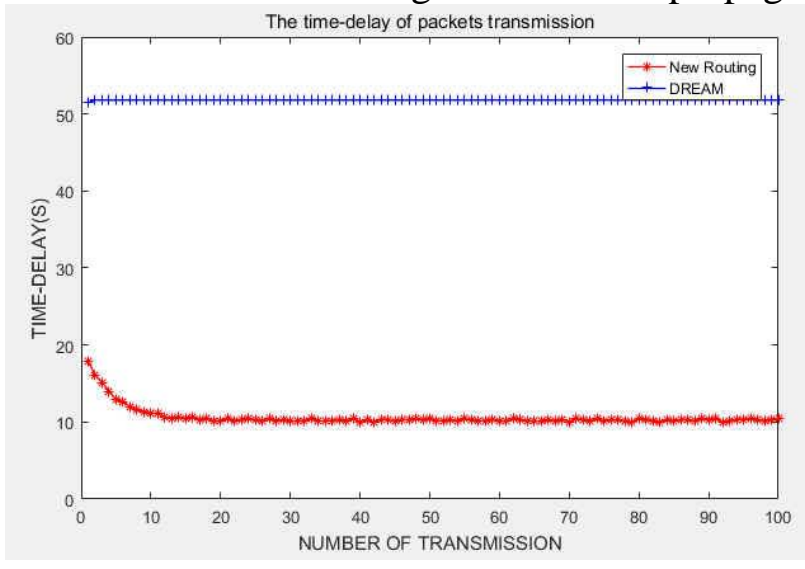

(a)Time-Delay

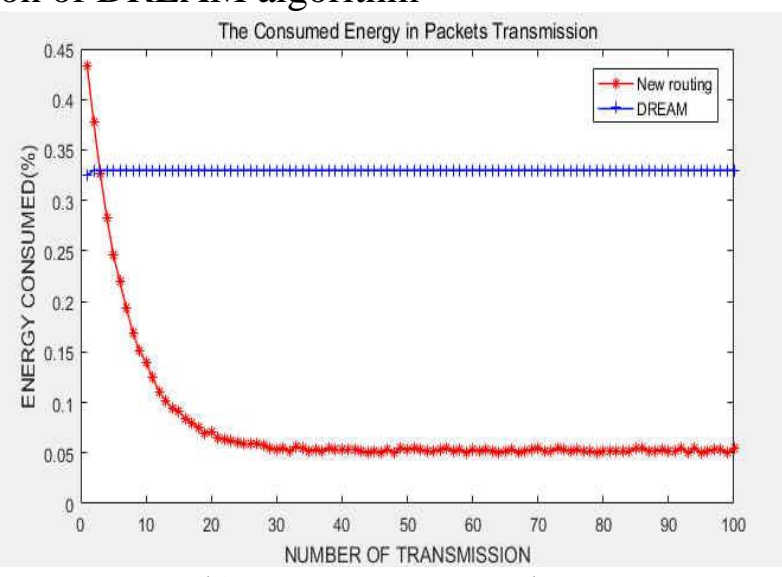

(b)Energy-consumed

Fig. 5 Performance comparison between the new algorithm and DREAM

DREAM does not keep a routing table, and send data packet by Directional flooding with nodes' geographic information each time. During the initial stage of packets transmission, the transmission delay of new packet will be increased due to the interference from old data packets in network. That is exactly the explanation of the previous 3-packet-propagations' delay gradually rising phenomenon. Subsequently, due to the load of the network has reached a stable value, the time-delay and energy-consumed in each transmission will be stable at a fixed value. In the whole experiment, the nodes that participate in the communication will be basically the same, so the loss of each energy will also be a fixed value.

\section{Conclusion}

In view of the situation that the deep sea acoustic network can not obtain the location information of the nodes, this paper proposes a new algorithm based on the mechanism of "gas circulation". The algorithm does not need the location information of nodes, and transmit packets at the same time with constructing a path. under the dual influence of pheromone and ant pheromone, the new algorithm 
will be more and more convergent to the current optimal path with the increase of the number of communication. Simulation results show that the new algorithm has excellent performance in reducing the time-delay, saving energy of network, and has a good application prospect in the large sparse deep sea acoustic network which can not be informed of nodes' geographical location information.

\section{Acknowledgements}

This paper was supported by the Natural Science Foundation of Naval Engineering University (No.HGDKYJGZX15007).

\section{References}

[1]. I F Akyildiz, D Pompili, T Melodia. Underwater Acoustic Sensor Networks: Research Challenges [J]. Ad Hoc Networks. Vol. 3(2005) No. 3, p. 257-279.

[2]. Hu HongNing, Liu Zhong, Yang Bin. BFDREAM: A new routing protocol for deep sea acoustic network[C]. 2010 IEEE 10th INTERNATIONAL CONFERENCE ON SIGNAL PROCESSING PROCEEDINGS (ICSP2010). Beijing: Springer Verlag.2010.p. 2377-2381.

[3]. Marco Dorigo, Thomas Stutzle. Ant Colony Optimization [M]. America, Cambridge: The MIT Press, 2004.p.24.

[4]. BILCHEV G, PARMEE I. The ant colony metaphor for searching continuous design spaces[C]. Proc of AISB Workshop on Evolutionary computing. London: Springer-Verlag. 1995. p.25-39.

[5]. WANG Lei, WU Qi-di. Ant system algorithm for optimization in continuous space [C].Proc of IEEE International Conference on control applications. 2001. p.395-400.

[6]. HU Xiao-min, ZHANG Jun, CHUANG H S H. SamACO: variable sampling ant colony optimization algorithm for continuous optimization [J]. IEEE Trans on systems, Man, and Cybernetics, Part B: Cybernetics. Vol.40 (2010) No.6, p. 1555-1566.

[7]. XIAO Jiang, LI Liang-ping. A hybrid ant colony optimization for continuous domains [J]. Expert Systems with Applications.Vol.38 (2011) No.9, p.11072-11077.

[8]. ZHANG Yong-de, HUANG Sha-bai. On ant colony algorithm for solving multiobjective optimization problems [J]. Control and Decision.Vol.20 (2005) No.2, p.170-173,178.

[9]. JIN Hao, LIU Weining. Ant colony algorithm for continuous function based on foraging-homing mechanism [J]. Computer Engineering and Applications. Vol.48 (2012) No.1, p.24-26.

[10]. Hu HongNing, Liu Zhong, Li Lu. A real-time directed routing protocol based on projection of convex holes on underwater acoustic networks [C]. The 3rd Internatioal Conference on Networks Security, Wireless Communications and Trusted Computing (NSWCTC2011). Wuhan. 2011. p.44-48.

[11]. Dorigo M, Gambardella L M. Ant Colony System: A cooperative learning approach to the traveling salesman problem [C].IEEE Transactions on Evolutionary Computation.1997.p.53-66. 